\title{
Ferroelectric Domain Structure of PZT Tetragonal Single Crystals Analysed by Scanning Probe Microscopy
}

\author{
J. A. Pérez*, I. K. Bdikin*, A. M. R. Senos*, P. Q. Mantas* and A. L. Kholkin* \\ *Department of Ceramics and Glass Engineering, CICECO, University of Aveiro, 3810-193 Aveiro, \\ Portugal \\ anamor@ua.pt
}

From the application point of view, the most interesting compositions in the lead zirconate titanate solid solutions, $\mathrm{PbZr}_{\mathrm{x}} \mathrm{Ti}_{1-\mathrm{x}} \mathrm{O}_{3}(\mathrm{PZT})$, are those near the centre of the phase diagram, where tetragonal and rhombohedral phases are separated by a boundary nearly independent on temperature. The composition of this so-called morphotropic phase boundary (MPB) is approximately 52/48 at room temperature. The piezoelectric coefficients, electromechanical coupling coefficients, dielectric permittivity and remnant polarization measured on ceramic samples reach a maximum in this region, not necessarily at the same composition [1], which explains why these compositions are technologically so interesting.

The maximum of the polarization in the vicinity of the MPB in polycrystalline materials has been explained by the coexistence of the rhombohedral and tetragonal phases, which increases the number of possible orientations for the polarization vector $\left(\mathrm{P}_{\mathrm{o}}\right)$, thus facilitating the domains reorientation [2]. The same result is obtained if a monoclinic phase appears between the other two, as proposed by Noheda et al. [3], because the presence of a monoclinic phase further increases the number of possible orientations for the polarization vector. Therefore, it is of great importance to study the domain distribution with the composition in PZT single crystal with chemical compositions near that of the MPB and try to complete the literature data [4-7].

PZT single crystals with a chemical composition in the tetragonal side of the phase diagram $(x=0.35$ and $\mathrm{x}=0.42$ ) were produced by a flux method, using $\mathrm{PbO}-\mathrm{KF}-\mathrm{PbCl}_{2}$, in the molar proportion of 2:1:2, and $4 \mathrm{wt} \%$ of $\mathrm{B}_{2} \mathrm{O}_{3}$ [8]. In this work, the piezoresponse force microscopy (PFM) was used to investigate the domains distribution in the PZT single crystals.

The structure of the domains was determined (Fig. 1a), b) e c)). The domain distribution found for each of these crystals is different and a decrease of the domain size with the increase of $\mathrm{Zr}$ content could be observed. Further work is needed to explain this behaviour. From a structural point of view, an opposite effect was expected, taking into account the decrease of the unit cell distortion upon approaching the MPB composition [9]. The role of possible extrinsic effects, such as defects or stresses, on the domain size should be considered.

The angle between the domains also increases with the $\mathrm{Zr}$ content, from $80^{\circ}$, for $\mathrm{x}=0.35$, to $90^{\circ}$, for $\mathrm{x}=0.46$. The angle of $80^{\circ}$ is not usual in tetragonal structures and may be related to reciprocal domains. 
Fig. 1d) shows that the local hysteresis loop $\left(d_{33}\right)$ for the $x=0.35$ PZT crystal is not symmetric. This effect denotes an asymmetry in the polarization of the crystal. Both crystals are mainly negatively orientated, as shown in Fig. 1d), where the peaks correspondent to the orientation of the polarization along the green line of Fig. 1a) are represented.

References:

[1] B. Jaffe, W.R. Cook, and H. Jaffe, Piezoelectric ceramics. London: Academic Press, 1971.

[2] V. A. Isupov, Soviet Physics Solid State, USSR. 10 (1968) 989.

[3] B. Noheda, D. E. Cox, G. Shirane, J. A. Gonzalo, L. E. Cross, S. E. Park, Appl. Phys. Lett., 74(14) (1999) 2059.

[4] E. I. Eknadiosiants, V. Z. Borodin, V. G. Smotrakov, V. V. Eremkin, A. N. Pinskaya, Ferroelectrics, 111 (1990) 283.

[5] K. Tsuzuki, K. Sakata, G. Ohara, M. Wada, Jpn. J. Appl. Phys., 12(10) (1973) 1500.

[6] T. Hatanaka, and H. Hasegawa, Jpn. J. Appl. Phys., Part 1, 31(9B) (1992) 3245.

[7] D. J. You, W. W. Jung, S. K. Choi, Y. S. Cho, Appl. Phys. Lett., 84(17) (2004) 3346.

[8] J. A. Perez, M. R. Soares, P. Q. Mantas, H. Amorin, M. E. V. Costa, A. M. R. Senos, Mats. Sci. Forum, vols. 514-516, (2006) 184.

[9] G. Arlt, and P. Sasko, J. Appl. Phys., 51(9) (1980) 4956.

The author J.A. Pérez gratefully acknowlwdges the financial support of the Portuguese Foundation for Science and Technology (FCT)
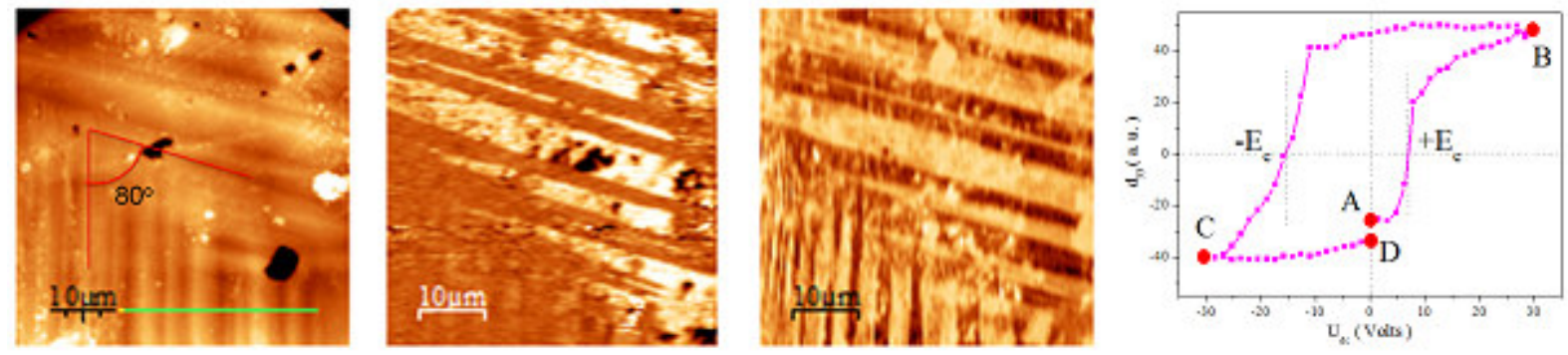

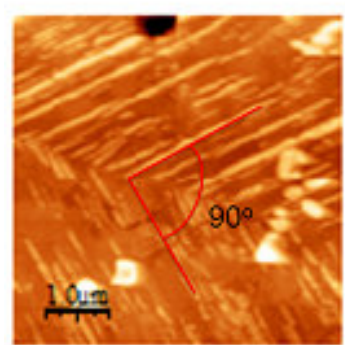

a)

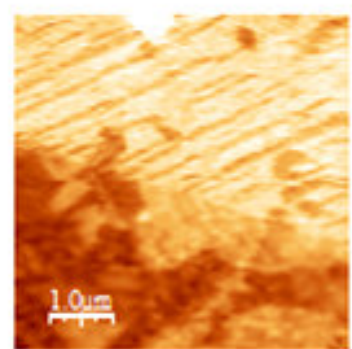

b)

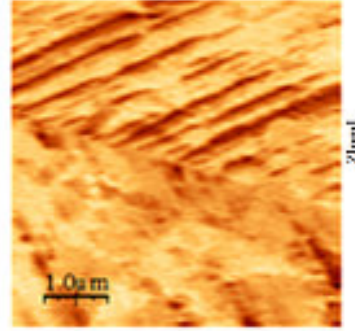

c)

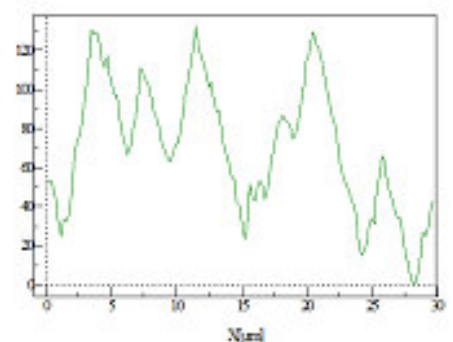

d)

Fig. 1. PFM images of the [100]-oriented PZT single crystals: (a) topography, (b) in-plane and (c) out-of-plane (OPP), in the top for $\mathrm{x}=0.35$ and bottom for $\mathrm{x}=0.42$; (d) local hysteresis loop $\left(\mathrm{d}_{33}\right)$ and cross section along the green line for the PZT single crystal with $\mathrm{x}=0.35$. 